\title{
Modelling bushfire impact on hydrology: The implications of the fire modelling approach on the climate change impact
}

\author{
$\underline{\text { G.R. Willgoose }}^{\mathrm{a}}$ \\ ${ }^{a}$ Discipline of Civil, Surveying and Environmental Engineering, Faculty of Engineering and the Built \\ Environment, The University of Newcastle, Callaghan, 2308 Australia \\ Email: garry.willgoose@newcastle.edu.au
}

In dry environments bushfire is an integral part of the dynamics of the environmental system. Thus any computational model of the environment (e.g. the effect of climate change on vegetation, hydrology, landform evolution, etc) needs to include a model of bushfire (e.g. Thonicke, et al 2001). Distributed models need to not only model the temporal distribution of bushfire but also their spatial distribution. The spatial properties of bushfires include the location of the initiation point of the fire and the spread in space from that initiation point. Thus the time history of bushfire at any specific location in space is a function of the rate of bushfires in the region surrounding that point, and the likelihood that any given fire will spread to the point under consideration. Thus the fire history at a point is a function of the both the temporal and spatial properties of bushfire.

In the applications contemplated in this paper we are making predictions into the future so we need to be able to make predictions in a statistical, rather than a deterministic, sense. Accordingly we are looking for a minimalist model that involves replication of the statistical properties of bushfire rather than a deterministic model that might be used for the prediction of the behavior of any individual bushfire event. Deterministic models of bushfire propagation exist (e.g. FARSITE; Finney et al, 1998) but their use for statistical prediction is limited because they require a variety of environmental inputs (e.g.. humidity, wind speed, fuel load) that themselves need to be independently predicted (Pastor et al, 2003).

This paper explores a simpler, purely statistical, approach where bushfire is modeled directly by monte-carlo simulation. The model is calibrated to published data for bushfire and then some preliminary assessment of its usefulness for looking at the impact of bushfire on hydrology is examined. The idea of the model presented here is that fire is modeled as a random process on a grid. Fire is randomly initiated at a time and a location, and some area around that location is then burnt. The burn history of the landscape is then the history of all of the individual fires that have been randomly generated in space and time.

A spatially distributed stochastic simulation bushfire model is presented and it is calibrated to remotely sensed fire data. It is shown that the assumption of how the frequency of a fire and its subsequent extent are related has a significant impact on the net impact of bushfire on hydrology. In particular, it highlights how important it is to distinguish between how often, on average, a bushfire burns a location, and how often, on average, a location has an ignition event. These two properties are different and that climate change predictions will need to provide information on both of these properties.

The fire model presented is very simple and this is its first test against field data. There are a number of areas where it might be improved to gain better fit to field data. The most obvious is a relationship between the last time a node was burnt and where fires are initiated and/or propagated. This will likely be linked to rate of recovery of biomass and thus there might be some form of climate dependency. There is also a need for more thorough testing of the spatial statistics of the model using data from other sites (e.g. Haydon et al 2000) and more comprehensive longitudinal datasets using NDVI and Landsat datasets.

Keywords: bushfire, hydrology, simulation, scaling, climate change 


\section{INTRODUCTION}

In dry environments bushfire is an integral part of the dynamics of the environmental system. Thus any computational model of the environment (e.g. the effect of climate change on vegetation, hydrology, landform evolution, etc) needs to include a model of bushfire (e.g. Thonicke, et al 2001). Distributed models need to not only model the temporal distribution of bushfire but also their spatial distribution. The spatial properties of bushfires include the location of the initiation point of the fire and the spread in space from that initiation point. Thus the time history of bushfire at any specific location in space is a function of the rate of bushfires in the region surrounding that point, and the likelihood that any given fire will spread to the point under consideration. Thus the fire history at a point is a function of the both the temporal and spatial properties of bushfire.

In the applications contemplated in this paper we are making predictions into the future so we need to be able to make predictions in a statistical, rather than a deterministic, sense. Accordingly we are looking for a minimalist model that involves replication of the statistical properties of bushfire rather than a deterministic model that might be used for the prediction of the behavior of any individual bushfire event. Deterministic models of bushfire propagation exist (e.g. FARSITE; Finney et al, 1998) but their use for statistical prediction is limited because they require a variety of environmental inputs (e.g.. humidity, wind speed, fuel load) that themselves need to be independently predicted (Pastor et al, 2003).

This paper explores a simpler, purely statistical, approach where bushfire is modeled directly by monte-carlo simulation. The model is calibrated to published data for bushfire and then some preliminary assessment of its usefulness for looking at the impact of bushfire on hydrology is examined. The idea of the model presented here is that fire is modeled as a random process on a grid. Fire is randomly initiated at a time and a location, and some area around that location is then burnt. The burn history of the landscape is then the history of all of the individual fires that have been randomly generated in space and time. The details of the model and its calibration follow.

\section{OBSERVED SCALING OF FIRE AREA AND FREQUENCY}

Data exist to suggest that a purely statistical representation of bushfire is in fact feasible.

Malamud et al (1998) observed a log-log scaling relationship between fire size and fire frequency for four regions in the USA and Australia (US Fire and Wildlife Service Lands; Western USA; Alaskan boreal forests; ACT, Australia) (see Figure 1). These relationships are log-log linear over a large range of fire area. The average fire size for a given frequency of recurrence is different for each region. However, the scaling between frequency and fire size (the slope of the fitted line in Figure 1) is surprisingly consistent suggesting the possibility of universal behaviour for frequency versus area. It is not clear from Malamud what role fire suppression activities (both before and during the fire events) had in determining the ultimate fire area. However, the $\log$-log linear scaling relationship provides a useful hypothesis that we will use in this paper to examine the effect of climate change and wildfire on hydrology in a stochastic modeling framework. Yates et al (2008) provide a fire frequency analysis for Northern Australia tropical savannas that also shows this log-log scaling.

\section{THE STOCHASTIC MODELLING FRAMEWORK}

The model as described below was implemented as a Python module, WildFire2, in the TelluSim environmental modeling system (Willgoose, 2009).

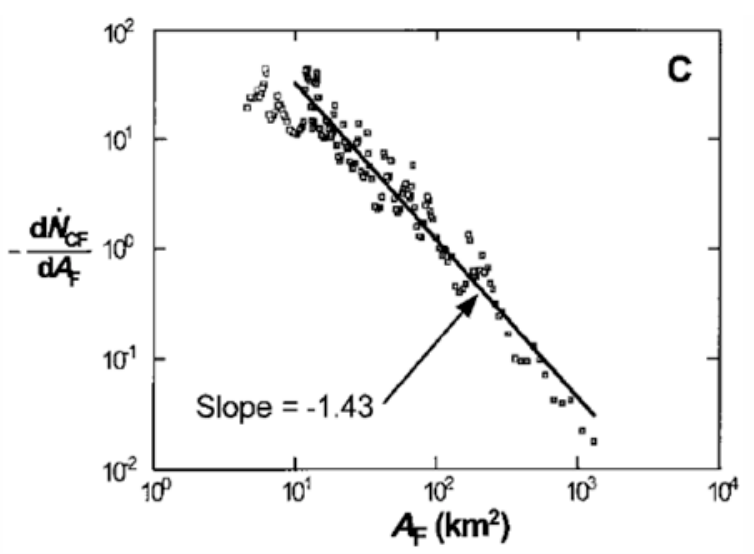

Figure 1: Typical fire frequency vs burnt area data from Malamud et al (1998). The vertical axis is the noncumulative number of fires per year with area $A_{F}$ 


\subsection{The Fire Model}

Fire is simulated with a monte-carlo model consisting of three parts; (1) A fire initiation event, (2) a location for the fire, and (3) the area for the fire.

The fire initiation event is function of the rate of occurrence of fires/unit area. Normally fire data does not measure this fire occurrence rate directly but rather it needs to be determined indirectly from the amount of land burnt in any year as measured from remote sensing images. The simplest mathematical case is that where it can be assumed that any point in the landscape can only be burnt once in any one year and where all fires are the same area. In that case the mean rate of fire occurrence/unit area/year $\lambda$ is simply

$\lambda=\frac{(\% \text { of area burnt } / \text { year })}{100 *(\text { mean area of each fire })}$

In the model a fire occurs in the domain in any given timestep if a uniformly distributed random number in the range $[0$, domain area] is greater than $\lambda$. In the simulations that follow a mean fire area is assumed and the rate determined by calibration to the field fire data.

The location of the fire is randomly located in space with a Poisson distribution. In the basic form of the model, used in this paper, there is no influence of the fire initiation point from the burn history of that point so that recently burnt points are just as likely to be initiation locations as other points.

The size and the shape of the fire can be modeled with a variety of mechanisms. Three size assumptions are tested in this paper (1) the burnt area of all fires have the same area, (2) the burnt areas are randomly distributed (with a uniform distribution) between a specified upper and lower bound, (3) the burn areas are a function of the frequency of occurrence as given by Malamud's data with scaling exponent $=-1.4$. Previous unpublished work by the author suggested that the influence of the shape of the fire on average burn statistics is second order so, for simplicity, in this paper we use only square burn areas.

For the case where the fire area varies with occurrence frequency (as in Malamud's data) the area range of fires to be simulated was discretized and the relative rate of occurrence for each size within that range applied from the scaling relationship. The total fire return rate was calibrated to the percent burnt data and the relative rates scaled up and down to match the percent burnt per year data. This ensures that the relative rate of fire occurrence for the different size is matched and the total number of fires/year matches the percent burnt data.

\subsection{The Hydrology Model}

Robust data about the effect of fire on hydrology and the timescales over which it recovers to pre-fire rates is rather sparse, site/geography/soil/vegetation dependent, and difficult to generalise. Some studies indicate that runoff rates increase post-fire because of the creation of seal on the soil surface by heat. Once this seal is broken runoff rates return to pre-fire rates quickly, perhaps within the year (e.g. Beeson et al., 2001). Other studies indicate long term declines in runoffs that take many years to recover (e.g. Kuczera, 1987). The driver for this latter behaviour is that trees use more water to replace biomass destroyed in the fire and this increase in biomass production requires increased water use (and consequent decreased runoff) through the link between transpiration and photosynthesis. This paper is concerned about reductions in water yield so the focus will be on the Kuczera curve. Kuczera presented data fit to catchment runoff for a number of catchments and found that yields declined for 20-30 years after the fire, then
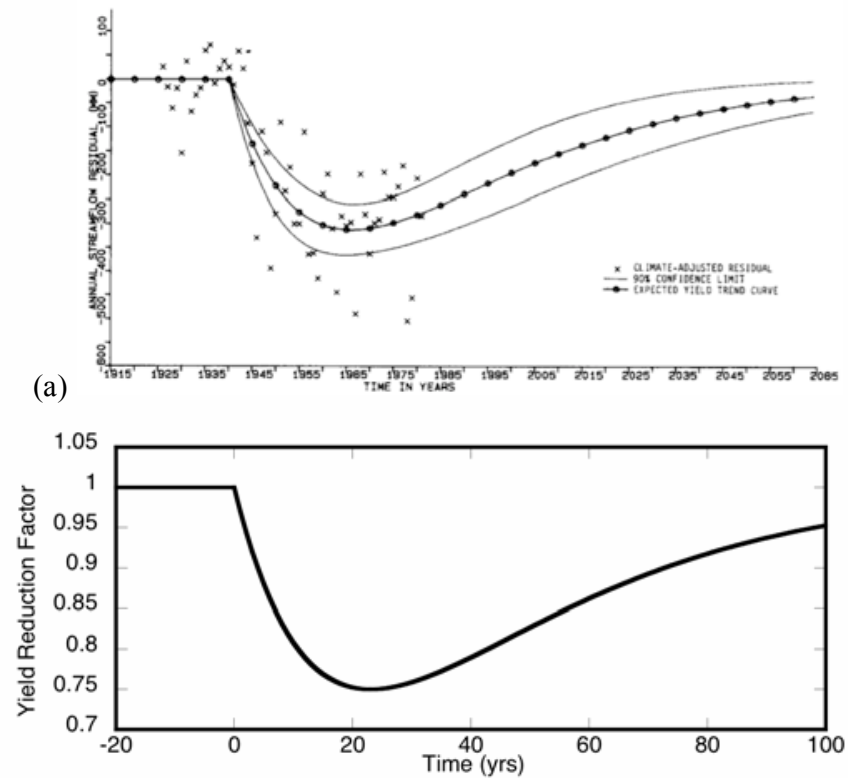

(b)

Figure 2: (a) Runoff yield reduction curve after fire from Kuczera (1987), (b) The yield curve from Equation (2). 
subsequently increased with time and that full recovery of pre-fire yield was projected to take over 100 years. The maximum runoff decline (occurring at 20-30 years) varied from $25-50 \%$ in catchments where $100 \%$ of the catchment was burnt. The model used in this paper for yield is based on Kuczera's findings but was not systematically fit to his datasets. The equation used here for the yield reduction is:

postfire yield $=$

prefire yield $\left(1-\left(1-e^{-\lambda_{1} t}\right) e^{-\lambda_{2} t}\right)$

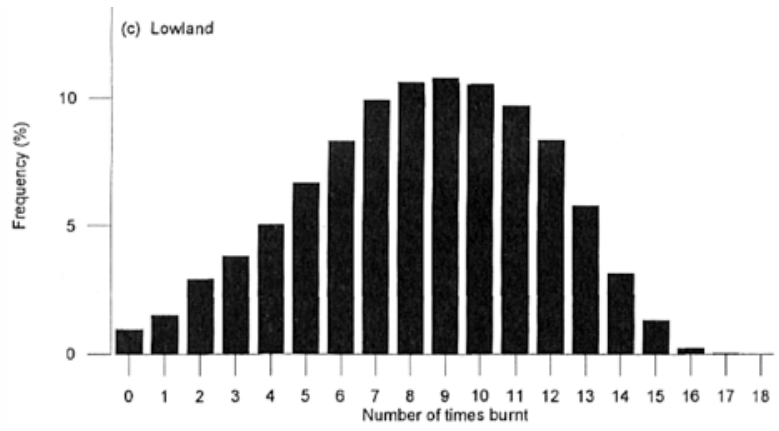

Figure 3: Pixel burn data from Russell-Smith (1997)

where $\lambda_{1}$ and $\lambda_{2}$ are both equal to 0.03 and $t$ is the number of years since the fire (Figure 2b). The curve in Figure 2a that equation (2) approximates is commonly called the Kuczera Curve.

Later in the paper we will also look at an accelerated Kuczera Curve where $\lambda_{1}$ and $\lambda_{2}$ are equal to 0.3 . This parameter change increases the rate of change of vegetation impact by a factor of 10 (or in Figure $2 \mathrm{~b}$ changes the time axis from 100 years to 10 years). This will be used to explore the impact of faster recovery of vegetation as might expected for shrubs and grasses, rather than the trees in the original Kuczera work.

\subsection{The Climate Change Model}

The role of climate change in the simulations here will be modeled in a very simple fashion. We will assume that the sole role of climate change will be to change the frequency of a given size fire event. In this way we will capture the idea that as the landscape becomes drier and fire fuel load is drier there will be an increase in fire initiation events.

It is recognized that this is extremely simplistic and ignores effects such as (1) the reduction in fuel load as the climate dries which in turn reduces vegetation growth and thus dead biomass (e.g. Bradstock, 2010) or (2) change in vegetation regime (Murphy et al, 2010). However, this would require a climate simulator to run a vegetation model, which is beyond the scope of this paper. This paper explores a simple approach to including fire occurrence in a spatially distributed hydrology model without the explicit use of climate data in much the same way that autoregressive moving average (ARMA) models have historically been used for single site stochastic hydrology simulation without the explicit use of climate data.

\section{CALIBRATION OF THE FIRE MODEL TO FIELD DATA}

\subsection{The Field Data}

Russell-Smith et al (1997) used remote sensing (Landsat Multispectral Scanner) to map the areas of individual fires in Kakadu National Park in the period 1980-1994. These data were ground-truthed with an

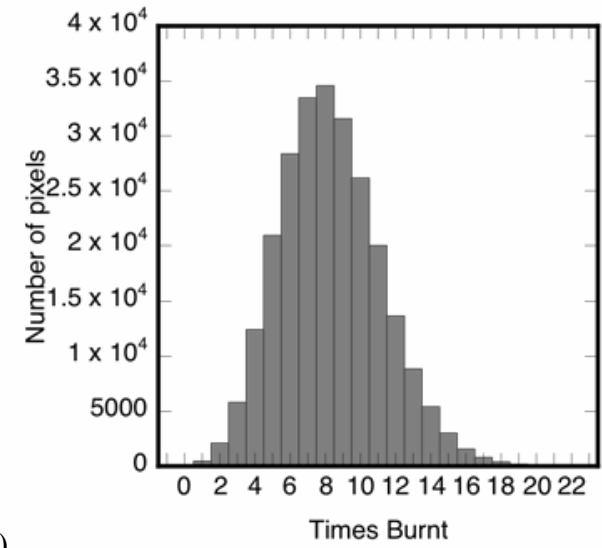

(a)

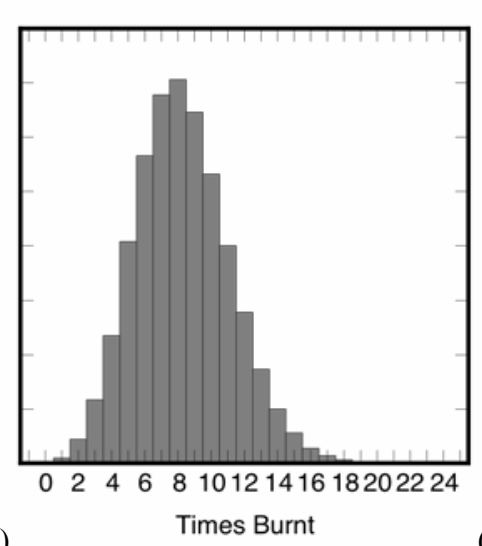

b)

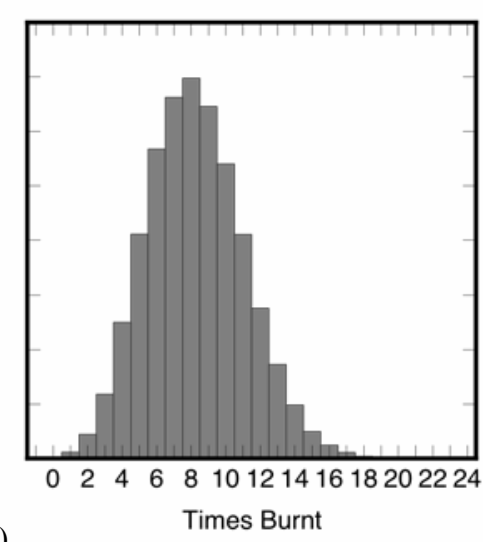

(c)

Figure 4: Calibration for the 3 burnt area models at 15 years. (a) Constant area $=100$ pixels, (b) Variable area from 0 to 200 pixels (average area $=100$ pixels), and (c) the Malamud scaling model with exponent $=-1.4$. 


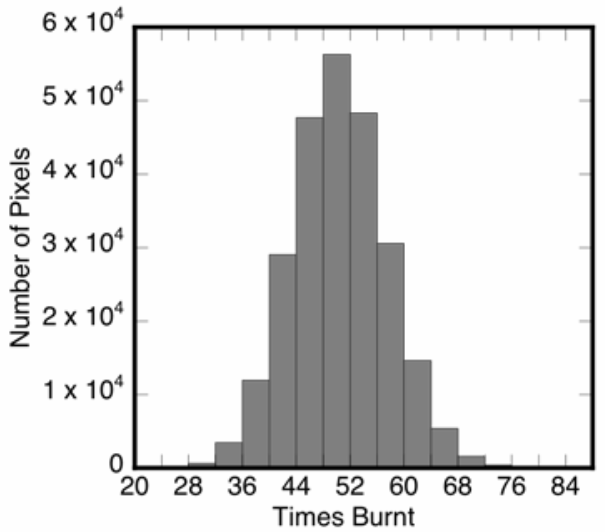

Figure 5: Calibrated constant area burn model after 90 years, starting from $100 \%$ unburnt at time $=0$.

Compare the symmetry with Figure $4 a$.

$80 \%$ agreement in burnt areas. This data was further disaggregated into the three major geographic zones in Kakadu; Floodplain, Arnhem Plateau and Lowlands (this paper only uses the Lowlands data, Figure 3). From these data they were able to derive statistics for:

- percentage area burnt each year,

- area burnt disaggregated by vegetation type,

- number of times burnt in 1980-1994 (Figure 3), and

- relationship between burnt and preceding wet season rainfall (most burning occurs in the dry season).

Yates et al (2008) and Murphy et al (2010) provide more restricted statistical analysis of fire scars in the same region and are quantitatively consistent where there is overlap in the statistical analyses used.

\subsection{Calibration}

The 3 different fire models were calibrated to the fire data of Russell-Smith by selecting a fire burn area and adjusting the fire initiation rate/unit area until the model gave the same mean number of times burnt (i.e. number of times burnt=8.3). The simulations started with a completely unburnt domain, effectively equivalent to the analysis of Russell-Smith. The probability distribution functions (pdfs) of the 3 fits are almost identical (Figure 4) with only insignificant differences between them. The pdfs of the calibration are all slightly skewed to the left while the field data is slightly skewed to the right. This appears to be a consistent characteristic of the model and is independent of burn shape, area and orientation. At very early times (when a large proportion of the region is still unburnt) the model is strongly skewed to the left and Russell-Smith did report one data set similarly skewed to the left. If the model is allowed to run for much longer times (i.e. beyond the 15 years of the calibration data) the model converges on a symmetric, unskewed pdf (Figure 5) but never achieves a pdf skewed to the right exhibited by the Lowlands dataset. This suggests that there may be an as yet unidentified 'age' effect in the skewness of the pdf.

Despite the similarity in the pdfs from the three models for the number of times burnt, the spatial patterns of the burnt areas are visually different (Figure 6) suggesting that spatial geostatistical measures (such as spatial correlation scales) of the burn pattern might be different. Russell-Smith did not report any geostatistics so no comparison has been made. (a)

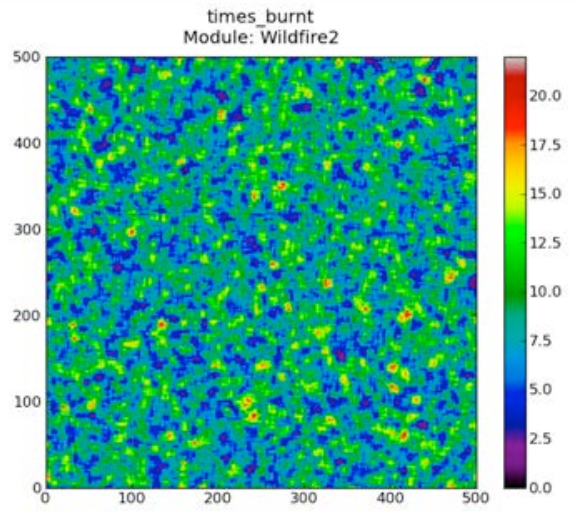

(b)

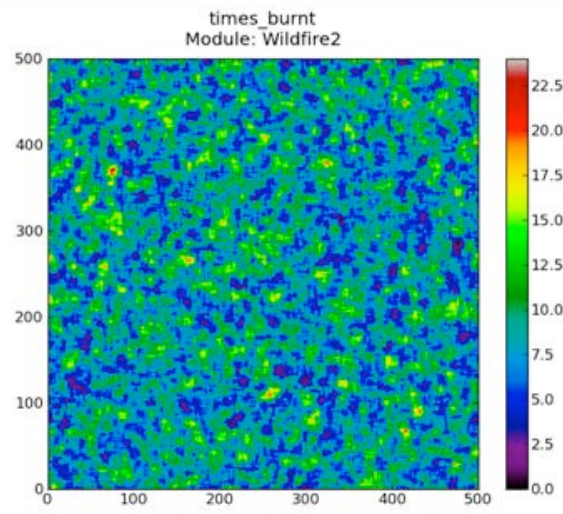

(c)

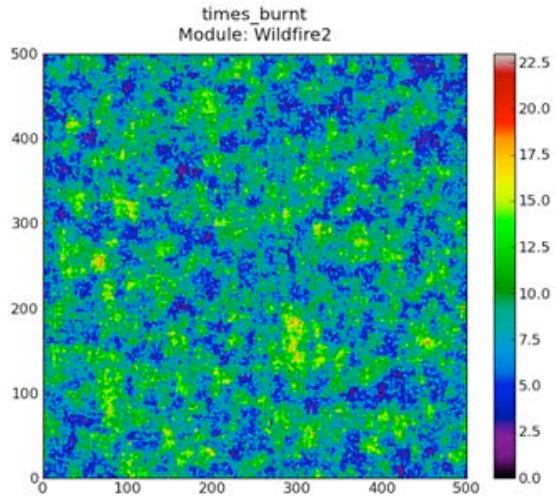

Figure 6: Burn patterns from the calibration for (a) constant area, (b) variable area, (c) Malamud scaling model. 


\section{THE HYDROLOGY IMPACT OF THE CHANGE IN FIRES DUE TO CLIMATE CHANGE}

\subsection{Baseline Fire Hydrology Simulations}

If a time burnt pdf (i.e. Figures 3-5) is available then a simple model for the effect on hydrology of the Kuczera Curve can be determined without the direct use of the monte-carlo model. Each ordinate of the pdf gives the number of times the catchment is burnt in a given period (in the case of Russell-Smith's data, the 15 years from 19801994) and from this the mean time since that portion of the catchment was last burnt can be determined. For instance, if $12 \%$ of the catchment was burnt 10 times in a 15 year monitoring period then on average $12 \%$ of the catchment will have been burnt 1.5 years previously. This is no doubt a simplification of the process, because it ignores:

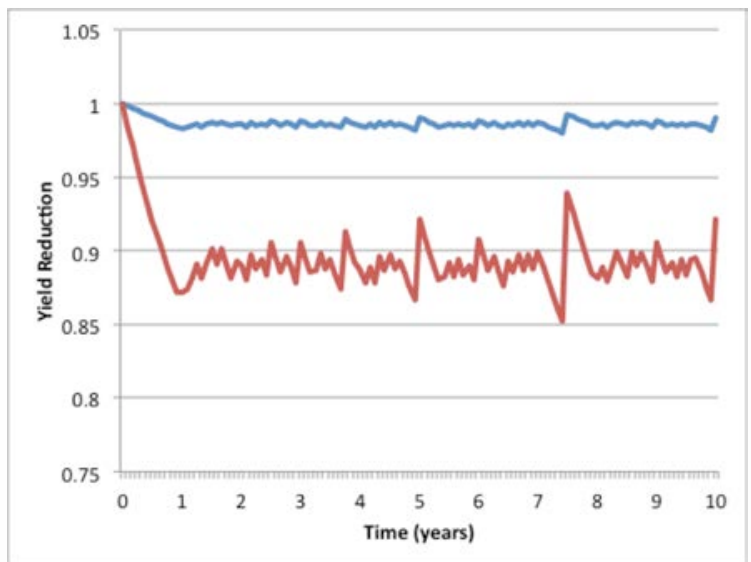

Figure 7: Simulated yield reduction for the normal (blue line) and accelerated Kuczera Curve (red line) under current climate starting from an unburnt landscape.

(1) the burn history prior to the monitoring period (the reason for the difference between Figure 4a and Figure 5) and,

(2) the cumulative impact of regions being burnt multiple times on hydrology (Kuczera indicates that yield reductions are cumulative but not in a well understood way).

That said it is a simple analytic solution that can be used to compare with model simulations. Figure 7 shows its application to the observed data in Figure 3 for a period of 10 years. The amounts of reduction in the hydrology should not be taken as predictions for a specific site because the fire data comes from a highly fire prone region in monsoonal Northern Australia and the Kuczera Curves comes from a much less fire prone region in alpine Southern Australia. Rather they are provided here as a simple demonstration of the technique. To explore what might be a more realistic response in the monsoonal tropics where most vegetation are grasses and recovery from fire is quick we use the accelerated Kuczera Curve (Figure 7). It can be seen that the relative impact of the acceleration of the timescales of vegetation response magnifies the reduction in the runoff yield, everything else being equal.

\subsection{Climate Change Hydrology Simulations}

To look at the incremental effect of climate change we cannot use the Russell Smith data but we must use the model calibrated to the data. In the discussion that follows only the Malamud scaling model is discussed because the results for all three models (constant area, variable area, Malamud scaling) are comparable in the results they yield for the incremental impact of climate change driven changes in fire frequency. The results
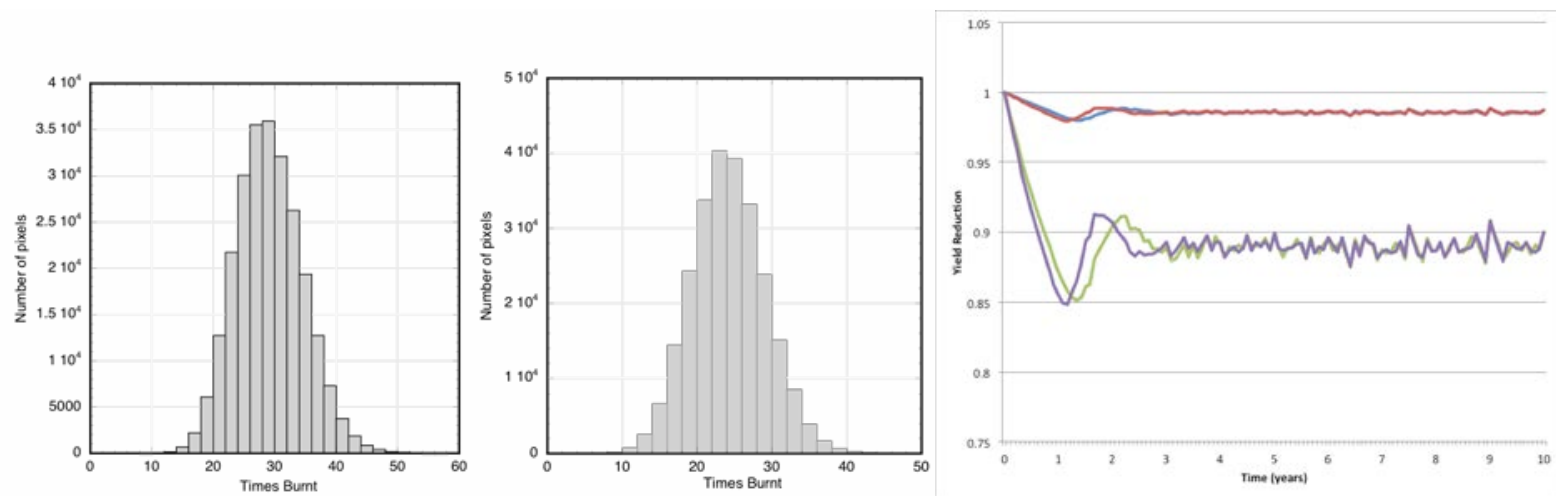

Figure 8: The effect of climate change on hydrology. (a) the pdf for the number of times burnt for the baseline case for a 45 year simulation, (b) as for figure (a) but with a $20 \%$ increase in the rate of fire frequency, (c) the effect on runoff yield using the traditional (red and blue) and accelerated (green and lilac) Kuczera curves for pre (blue and green) and post (red and lilac) 20\% climate change. 
are shown in Figure 8.

The surprising result is that climate change driven bushfire changes have almost no impact on the hydrology with the yields after the first few years being almost identical. The main difference is in the timing of the initial change in hydrology with a slightly faster response after climate change (due to the higher frequency of fires) but both systems settle down to a long average drop in runoff yield that is almost identical. Given we have applied a step change in climate and climate change is in fact gradual the differences in the first few years are unlikely to be noticeable in practice. The preliminary conclusion is that climate change, while changing bushfire frequency, will not produce a measureable impact on runoff yield as a result of changes in fire frequency.

\section{CONCLUSIONS}

This paper has presented a new stochastic model for bushfire and demonstrated its use in spatially distributed hydrology simulation, and possible hydrological impacts of climate change driven changes in bushfire frequency. The analysis demonstrates that it might be a useful tool that could lead to novel insights into the environmental impact bushfire.

The fire model presented is very simple and this is its first test against field data. There are a number of areas where it might be improved to gain better fit to field data. The most obvious is a relationship between the last time a node was burnt and where fires are initiated and/or propagated. This will likely be linked to rate of recovery of biomass and thus there might be some form of climate dependency. There is also a need for more thorough testing of the spatial statistics of the model using data from other sites (e.g. Haydon et al 2000) and more comprehensive longitudinal datasets using NDVI and Landsat datasets.

\section{ACKNOWLEDGMENTS}

The author was supported by an ARC Australian Professorial Fellowship.

\section{REFERENCES}

Beeson, P. C., S. N. Martens, and D. D. Breshears (2001), Simulating overland flow following wildfire: mapping vulnerability to landscape disturbance, Hydrological Processes, 15 (5), 2917-2930.

Bradstock, R. A. (2010), A biogeographic model for fire regimes in Australia: current and future implications, Global Ecology and Biogeography, 19(2), 145-158.

Finney, M. A. (1998), FARSITE: Fire Area Simulator-Model Development and Evaluation, Rep. RMRSRP-4, USDA Forest Service Rocky Mountain Research Station.

Haydon, D. T., J. K. Friar, and E. R. Pianka (2000), Fire-driven dynamic mosaics in the Great Victoria Desert, Australia - II. A spatial and temporal landscape model, Landscape Ecology, 15(5), 407-423.

Kuczera, G. (1987), Prediction of Water Yield Reductions Following a Bushfire in Ash-Mixed Species Eucalypt Forest, Journal of Hydrology, 94(3-4), 215-236.

Malamud, B. D., G. Morein, and D. L. Turcotte (1998), Forest fires: An example of self-organized critical behavior, Science, 281(5384), 1840-1842.

Murphy, B., J. Russell-Smith, and L. Prior (2010), Frequent fires reduce tree growth in northern Australian savannas: implications for tree demography and carbon sequestration, Global Change Biology, 16(1), 331343.

Pastor, E., L. Zarate, E. Planas, and J. Arnaldos (2003), Mathematical models and calculation systems for the study of wildland fire behaviour, Progress in Energy and Combustion Science, 29(2), 139-153.

Russell-Smith, J., P. G. Ryan, and R. Durieu (1997), A LANDSAT-MSS derived fire history of Kakadu National Park, monsoonal northern Australia, 1980-94: seasonal extent, frequency and patchiness, Journal of Applied Ecology, 34, 748-766.

Thonicke, K., S. Venevsky, S. Sitch, and W. Cramer (2001), The role of fire disturbance for global vegetation dynamics: coupling fire into a Dynamic Global Vegetation Model, Global Ecology and Biogeography, 10(6), 661-677.

Yates, C. P., A. C. Edwards, and J. Russell-Smith (2008), Big fires and their ecological impacts in Australian savannas: size and frequency matters, International Journal of Wildland Fire, 17(6), 768-781.

Willgoose, G. R. (2009), TELLUSIM: A Python Plug-in Based Computational Framework for Spatially Distributed Environmental and Earth Sciences Modelling, 18th World IMACS / MODSIM Congress, Cairns, Australia, 13-17 July 2009. 